\title{
A novel class of small RNAs: tRNA-derived RNA fragments (tRFs)
}

\author{
Yong Sun Lee, ${ }^{1,2}$ Yoshiyuki Shibata, ${ }^{1}$ Ankit Malhotra, and Anindya Dutta ${ }^{3}$ \\ Department of Biochemistry and Molecular Genetics, University of Virginia, Charlottesville, Virginia 22908, USA
}

\begin{abstract}
New types of small RNAs distinct from microRNAs (miRNAs) are progressively being discovered in various organisms. In order to discover such novel small RNAs, a library of 17- to 26-base-long RNAs was created from prostate cancer cell lines and sequenced by ultra-high-throughput sequencing. A significant number of the sequences are derived from precise processing at the $5^{\prime}$ or $3^{\prime}$ end of mature or precursor tRNAs to form three series of tRFs (tRNA-derived RNA fragments): the tRF-5, tRF-3, and tRF-1 series. These sequences constitute a class of short RNAs that are second most abundant to miRNAs. Northern hybridization, quantitative RT-PCR, and splinted ligation assays independently measured the levels of at least 17 tRFs. To demonstrate the biological importance of tRFs, we further investigated tRF-1001, derived from the 3' end of a Ser-TGA tRNA precursor transcript that is not retained in the mature tRNA. tRF-1001 is expressed highly in a wide range of cancer cell lines but much less in tissues, and its expression in cell lines was tightly correlated with cell proliferation. siRNAmediated knockdown of tRF-1001 impaired cell proliferation with the specific accumulation of cells in G2, phenotypes that were reversed specifically by cointroducing a synthetic $2^{\prime}$-O-methyl tRF-1001 oligoribonucleotide resistant to the siRNA. tRF-1001 is generated in the cytoplasm by tRNA 3'-endonuclease ELAC2, a prostate cancer susceptibility gene. Our data suggest that tRFs are not random by-products of tRNA degradation or biogenesis, but an abundant and novel class of short RNAs with precise sequence structure that have specific expression patterns and specific biological roles.
\end{abstract}

[Keywords: Small RNA; tRNA; deep sequencing; cancer cell proliferation]

Supplemental material is available at http://www.genesdev.org.

Received June 28, 2009; revised version accepted September 24, 2009.

Small noncoding regulatory RNAs have emerged as important players in diverse aspects of biology. The most extensively studied among them are microRNAs (miRNAs) that regulate target mRNA post-transcriptionally in animals and plants (for review, see Bartel 2004; Du and Zamore 2005; Lee and Dutta 2009). Another class of small RNA closely related to miRNAs is siRNA, a key intermediate in the RNAi pathway (Elbashir et al. 2001b). In plants, naturally occurring siRNAs such as tasiRNA (trans-acting siRNA) and natsiRNA (natural antisense transcripts siRNA) have been discovered (for review, see Chapman and Carrington 2007). In flies and mammals, endogenous siRNAs were reported only recently (for review, see Nilsen 2008), while synthetic dsRNA and siRNA have been used as a popular tool to knock down genes in animals for years.

A number of other small RNAs have also been identified in various organisms (for review, see Farazi et al.

\footnotetext{
${ }^{1}$ These authors contributed equally to this work.

${ }^{2}$ Present address: Department of Biochemistry and Molecular Biology,

University of Texas Medical Branch, Galveston, TX 77555, USA.

${ }^{3}$ Corresponding author.

E-MAIL ad8q@virginia.edu; FAX (434) 924-5069.

Article is online at http://www.genesdev.org/cgi/doi/10.1101/gad.1837609.
}

2008), including tncRNA (tiny noncoding RNAs), 21URNA, rasiRNA (repeat-associated siRNA), hcRNA (heterochromatic small RNA), scnRNA (scan RNA), piRNA (piwi-interacting RNA), tiRNA (transcription initiation RNA) (Taft et al. 2009), and PASR/TASR (promoter/ termini-associated sRNA) (Kapranov et al. 2007). Although some of these have been shown to play biological roles in processes such as heterochromatin formation and transposon silencing, most of them were discovered recently and are not fully understood. We believe that additional small RNAs await discovery, especially through the application of high-throughput sequencing technology (for review, see Morozova and Marra 2008).

In this study, we analyzed the global expression profile of small RNA (small RNome) in human prostate cancer cell lines by 454 deep sequencing. Next to miRNAs, the most abundant group of small RNA was fragments derived from tRNA (termed here as tRNA-derived RNA fragments [tRFs]). Besides their well-known role in translation, tRNAs have been shown to function in other cellular processes, such as reverse transcription, porphyrin biosynthesis, etc. (for review, see Giege 2008). Here, we identify a new aspect of tRNA biology. The characteristic and abundant expression of individual tRFs reported here, 
as well as their precise sequence composition, indicates that tRFs are not random degradation intermediates during tRNA biogenesis and turnover, but constitute a novel class of small RNA that deserves further scrutiny. One member of tRFs is shown to be required for cell viability.

\section{Results}

Cloning, identification, and characterization of $t R F-5, t R F-3$, and $t R F-1$ series

Small RNAs of 17-26 nucleotides (nt) were cloned from two prostate cancer cell lines, LNCaP and C4-2 (see the Supplemental Material for details), and sequenced by 454 deep sequencing technology (YS Lee, A Malhotra, D Sun, HK Kim, M Matecic, C Evans, RV Jensen, and A Dutta, in prep.). We obtained a total of 658,068 sequence reads, which are compared with one another to yield unique sequences and their cloning frequencies (Fig. 1). After extracting miRNAs that occupy $\sim 40 \%$ of total sequence reads, the remaining sequences (nonmicro-small RNA; nmsRNA) were subject to BLAST search against the human genome and EST sequences. Two mismatches were allowed to cover nmsRNA sequences that may have sequencing errors or post-synthesis RNA modifications, such as RNA editing.

We focused on 30 sequences that were abundantly cloned (>300 times) (Table 1). In comparison, a wellknown miRNA with a demonstrated biological function, miR-18a, was cloned 473 times, while a highly abundant miRNA, let-7a, was cloned 17,856 times. Of 695 miRNAs in the database (miRBase; http://microrna.sanger.ac.uk), a majority of them (635 miRNAs) were cloned $<200$ times. Each of these abundant nmsRNA sequences was further identified by examining its genomic locus on the University of California at Santa Cruz (UCSC) genome browser. Twenty-eight sequences were mappable to the human genome, in many cases at multiple loci (Fig. 2A).
Six of these are located within known transcripts and snoRNA (small nucleolar RNA), from which the small RNAs are likely to be derived. Of note, more than half of the abundant nmsRNA sequences mapped to tRNA loci (Fig. 2A; Table 1). Given that a cloning frequency approximately reflects the intracellular level of a small RNA, these tRNA-related small RNAs are expected to be more abundant than most miRNAs.

Primary transcripts (pre-tRNA) for mature tRNA are transcribed by RNA polymerase III and contain a 5' leader sequence and a $3^{\prime}$ trailer sequence that are trimmed during tRNA maturation (for review, see Morl and Marchfelder 2001; Hopper and Phizicky 2003). For most eukaryotic tRNAs, a CCA sequence is enzymatically added to the $3^{\prime}$ end of the trimmed tRNA intermediate. Among the 17 tRNA-related small RNAs, five and eight sequences are precisely aligned to the $5^{\prime}$ and $3^{\prime}$ ends of mature tRNA, respectively (Fig. 2B,C, tRF-5 and tRF-3 series). The $3^{\prime}$ end sequence of all eight tRF-3 RNAs includes the CCA that is post-transcriptionally added to the $3^{\prime}$ end of tRNAs during tRNA maturation. Four small RNAs of the tRF-1 series are located within pre-tRNA, and their $5^{\prime}$ ends start precisely after the $3^{\prime}$ ends of the mature tRNA sequence. The precise sequence conservation of the ends of all of the clones strongly suggests that a precise cleavage event is necessary for the generation of these tRFs.

To obtain a more comprehensive list of tRFs, we expanded our analysis to all small RNA sequences cloned more than five times in the library (see Fig 3A; Supplemental Table S2). Out of 1541 nmsRNA sequences that mapped to the human genome, $\sim 40 \%$ mapped to genomic loci containing a tRNA and flanking $25 \mathrm{nt}$ (Fig. 3A). Seventy-seven percent of these were identified as tRFs by their precise start and end sites, while the remaining $\sim 23 \%$ match tRNA at random locations in the body of the tRNA genes. The distributions of the $5^{\prime}$ (Supplemental Fig. S1A) and 3' (Supplemental Fig. S1B) ends of all
Figure 1. Pipeline of analyses of 454 deep sequencing data. A schematic of the 454 sequencing data analyses is depicted.

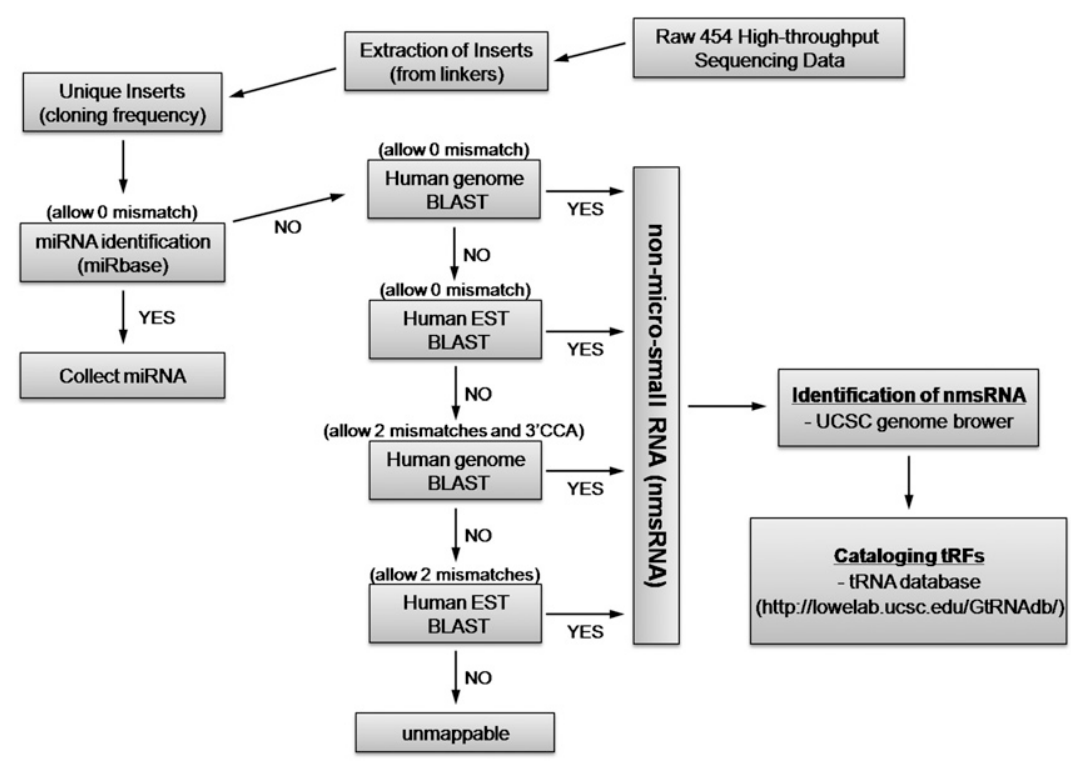


Table 1. List of abundantly cloned tRFs

\begin{tabular}{|c|c|c|c|c|c|}
\hline Name & Sequence & Length & $\begin{array}{l}\text { Cloning frequency } \\
\text { (sequence reads) }\end{array}$ & \multicolumn{2}{|c|}{ tRNA mapped } \\
\hline tRF-1001 & GAAGCGGGTGCTCTTATTT & $19 \mathrm{nt}$ & 3995 & tRNA-Ser-TGA & $33^{\prime}$ out \\
\hline tRF-1002 & GCCGGGTACTTTCGTATTTT & $20 \mathrm{nt}$ & 1090 & tRNA-Asp-GTC & 3' out \\
\hline tRF-1003 & GCTAAGGAAGTCCTGTGCTCAGTTT & $25 \mathrm{nt}$ & 378 & tRNA-Ser-GCT & $3^{\prime}$ out \\
\hline tRF-1004 & GTGTGTAGCTGCACTTTT & $18 \mathrm{nt}$ & 362 & tRNA-Asp-GTC & $3^{\prime}$ out \\
\hline tRF-3001 & ATCCCACCGCTGCCACCA & $18 \mathrm{nt}$ & 2852 & tRNA-Leu-TAG & $3^{\prime}$ \\
\hline tRF-3002 & ACCCTGCTCGCTGCGCCA & $18 \mathrm{nt}$ & 672 & tRNA-Ser-TGA & $3^{\prime}$ \\
\hline tRF-3003 & TCCCCGGCACCTCCACCA & $18 \mathrm{nt}$ & 657 & tRNA-Ala-AGC & $3^{\prime}$ \\
\hline tRF-3004 & TCCCCGGCATCTCCACCA & $18 \mathrm{nt}$ & 775 & tRNA-Ala-TGC & $3^{\prime}$ \\
\hline tRF-3005 & ATCCTGCCGACTACGCCA & $18 \mathrm{nt}$ & 1219 & tRNA-Ser-AGA & $3^{\prime}$ \\
\hline tRF-3006 & TCGATTCCCGGCCCATGCACCA & $22 \mathrm{nt}$ & 358 & tRNA-Gly-GCC & $3^{\prime}$ \\
\hline tRF-3007 & TCGATTCCCGGCCAACGCACCA & $22 \mathrm{nt}$ & 749 & tRNA-Gly-TCC & $3^{\prime}$ \\
\hline tRF-3008 & GTCCCACCAGAGTCGCCA & $18 \mathrm{nt}$ & 308 & tRNA-Arg-TCT & $3^{\prime}$ \\
\hline tRF-5001 & GACGAGGTGGCCGAGTGG & $18 \mathrm{nt}$ & 1797 & tRNA-Ser-GCT & $5^{\prime}$ \\
\hline tRF-5002 & TCCCTGGTGGTCTAGTGGTTA & $21 \mathrm{nt}$ & 906 & tRNA-Glu-CTC & $5^{\prime}$ \\
\hline tRF-5003 & GGTAGCGTGGCCGAGCGGTC & $20 \mathrm{nt}$ & 710 & tRNA-Leu-TAG & $5^{\prime}$ \\
\hline tRF-5004 & GCGTTGGTGGTATAGTGGT & $19 \mathrm{nt}$ & 1271 & tRNA-Gly-TCC & $5^{\prime}$ \\
\hline tRF-5005 & GCATGGGTGGTTCAGTGGTA & $20 \mathrm{nt}$ & 1221 & tRNA-Gly-GCC & $5^{\prime}$ \\
\hline
\end{tabular}

\begin{tabular}{lr}
\hline miRNA & Sequence reads \\
\hline miR-18a & 473 \\
let-7a & 17,856 \\
635 miRNAs & $<200$ \\
60 miRNAs & $>200$ \\
\hline
\end{tabular}

Seventeen tRFs are displayed with their sequence, length (in nucleotides), cloning frequency (number of sequence reads in 454 deep sequencing data), and relative location in the corresponding tRNA (see also the text and Fig. 2B,C). For comparison, we included sequence reads of two miRNAs, miR-18a and let-7a, and the number of miRNAs cloned more than or less than 200 times.

short RNAs along mature tRNA exhibit a significant enrichment of tRF-5-type and tRF-3-type molecules, suggesting that these are not random by-products of the degradation of tRNAs.

Presumably, tRF-5 and tRF-3 series are derived from mature tRNA by an endonucleolytic cleavage or exonuclease digestion. From the 454 sequencing data, we examined their nucleotide distribution at cleavage sites (Fig. 3B) and length distribution (Supplemental Fig. S2). $\mathrm{A}$ is enriched at the $3^{\prime}$ end of tRF- 5 series $(-1$ position of tRF-5 in Fig 3B). Cleavage sites for tRF-3 series are preferentially located between $\mathrm{A} / \mathrm{U}$ and $\mathrm{A} / \mathrm{U}$. The lengths of tRF-5 series exhibit a pattern of normal distribution in the size range of 15-25 nt (Supplemental Fig. S2A), which is similar to the size selection during our small RNA cloning. In contrast, the size range of tRF-3 series is skewed toward a smaller range of 13-22 nt (Supplemental Fig. S2B). Intriguingly, tRF-3 members of $20 \mathrm{nt}$ are specifically depleted. The preferences in nucleotide composition at cleavage sites and in size distribution of the tRFs, especially the tRF-3 series, further support the notion that they are not generated randomly.

The fraction of tRF-1 series $(14.2 \%)$ is lower than that of tRF-3 $(44.8 \%)$ or tRF-5 series $(41.0 \%)$ (Fig. $3 \mathrm{~A})$. The lower frequency of tRF-1 clones is explained by the hypothesis that tRF-1 sequences are released by 3' cleavage of the tRNA from the pre-tRNA. Of the 622 tRNA loci in the human genome (http://lowelab.ucsc.edu/ GtRNAdb), the distance between the 3 ' end of the mature tRNA and the termination signal of the RNA polymerase
III is variable (Supplemental Fig. S3). Only $14.6 \%$ of the tRNA loci could give rise to tRF-1 of the 16- to 27-base size range that was included in our cloning (Supplemental Fig. S3A), accounting for the lower yield of tRF-1 series. The size distribution of tRF-1-type molecules cloned did not correlate perfectly with the theoretical distribution expected if the cleavage of all pre-tRNAs gave rise to tRF-1 molecules (Supplemental Fig. S3B). This variance suggests that certain tRF-1-type molecules are selectively processed or retained in the cell for specific biological functions.

Thus, tRF-5, tRF-3, and tRF-1 (Supplemental Table S2) constitute the most abundant class of short RNAs in human prostate epithelial cells, second only to miRNAs. In addition, their precise start and end sites at or near the tRNA ends, together with their nonrandom property in size and nucleotide composition at cleavage junctions, strongly suggest that the tRFs are derived from tRNA cleavage in a specific manner.

\section{Expression of tRFs validated by other techniques}

Northern hybridization detected eight tRFs tested (Supplemental Fig. S4A,C). Not only did we detect a band of the correct size, but other bands in the range of 80-100 nt were apparent and could be precursors to the final tRFs. For tRF-1001, tRF-1002, and tRF-1003, the larger bands likely include the pre-tRNA from which the tRF is derived. For tRF- 5 and tRF-3 series, the probe also detects the corresponding mature tRNA, which is present at 
Lee et al.

A

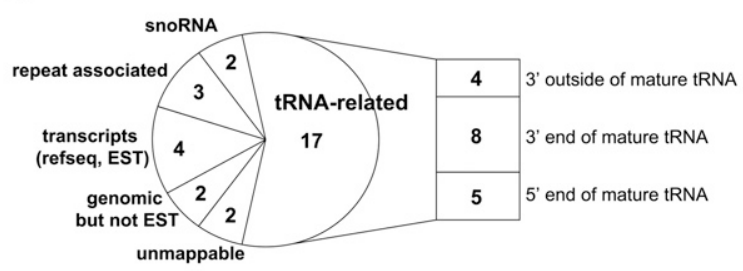

B

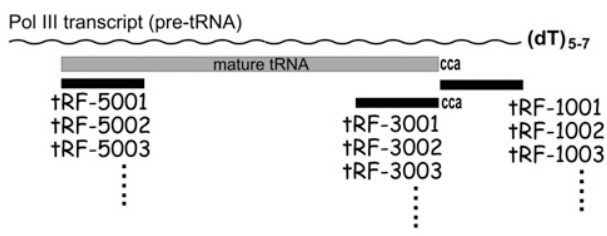

C

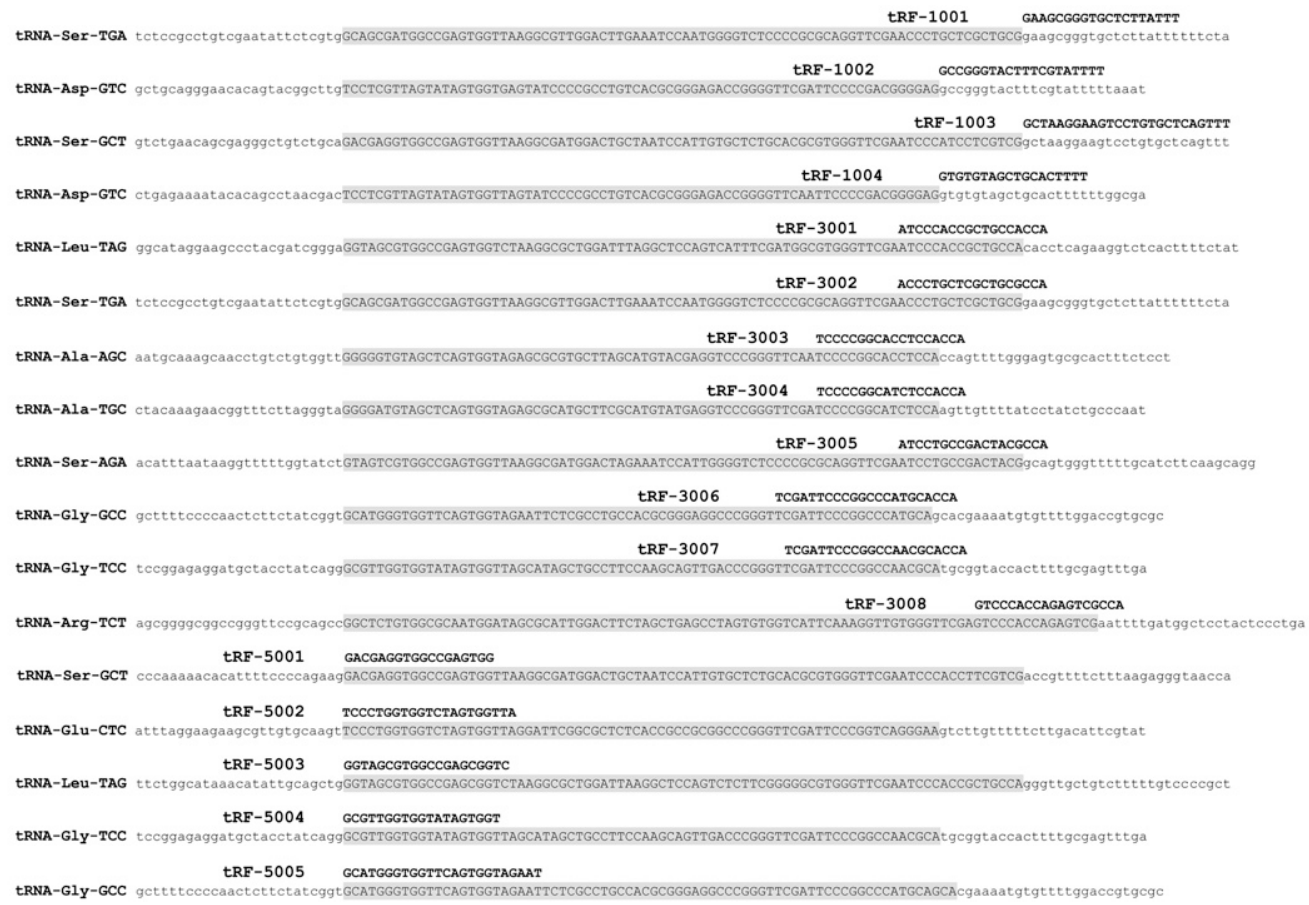

Figure 2. Classification and nomenclature of tRFs that were revealed by analysis of highly abundant nmsRNAs. $(A)$ Identification and classification of 30 nmsRNA sequences that were cloned abundantly. The nmsRNAs are grouped by their identity in the pie chart. (unmappable) nmsRNAs unidentifiable in the human genome sequence or the human EST database, probably due to as yet unreported splicing or sequence editing events. $(B, C)$ tRNA-related small RNAs (tRFs) are further classified by their relative location in the tRNA pretRNA. (B) Diagram of tRFs aligned at a tRNA locus (see $C$ for actual sequence alignment). (Wavy line) The genomic sequence for pre-tRNA ending with an oligo-dT stretch, the 3'-termination signal of RNA polymerase III. (Gray bar) A mature tRNA after 3' trimming of the pretRNA and addition of the CCA that is not present in the genomic sequence. (Black bars) Three groups of tRFs (tRF-5, tRF-3, and tRF-1 series) are aligned with the pre-tRNA or mature tRNA. tRFs are numbered, with the first digit indicating the group and the other digits forming a serial number in that group. $(C)$ Each of the 17 tRF sequences in $A$ (bold letters; see also Table 1) is aligned to the relative position (see also $B$ ) in the corresponding mature tRNA (shaded capital letters) or the flanking genomic regions (lowercase letters).

a much higher abundance than the tRF. Below the mature tRNA bands, there are additional bands of intermediate size (35-60 nt long) that could correspond to the tRNA cleavage products that were reported recently (Lee and Collins 2005; Haiser et al. 2008; Jochl et al. 2008; Kawaji et al. 2008; Li et al. 2008; Thompson et al. 2008; Zhang et al. 2009).

tRF-1001 was also measured by splinted ligation assays (Supplemental Fig. S4B; Maroney et al. 2008). This assay depends on ligation between the 3' end of tRF-1001 and the $5^{\prime}$ end of a ligation oligo, mediated through accurate annealing to a bridge oligo. Therefore, this assay detects RNA molecules with an accurate $3^{\prime}$-end sequence and is more specific in measuring the precursor tRNA transcript for tRF-1001. The tRF-1001 result was consistent with the Northern result (Supplemental Fig. S4, cf. A and B). It is worth pointing out that the levels of tRF-1001 and its precursor are not always perfectly correlated (e.g., C4-2 cells in Supplemental Fig. S4B), suggesting that the processing or stability of tRF-1001 may be regulated. Two tRF-3 series members, tRF-3001 and tRF-3009, were also measured by this assay (Supplemental Fig. S4D).

The expression levels of some tRFs were too low to be detected by Northern hybridization or splinted ligation assay, particularly for tRF-3 and tRF-5 series, where a strong signal from the mature tRNA and intermediate bands interferes with the tRF signal. To circumvent this problem, we used quantitative RT-PCR (qRT-PCR) detection in a method similar to the detection of miRNAs (for review, see Schmittgen et al. 2008). We gel-purified 
A

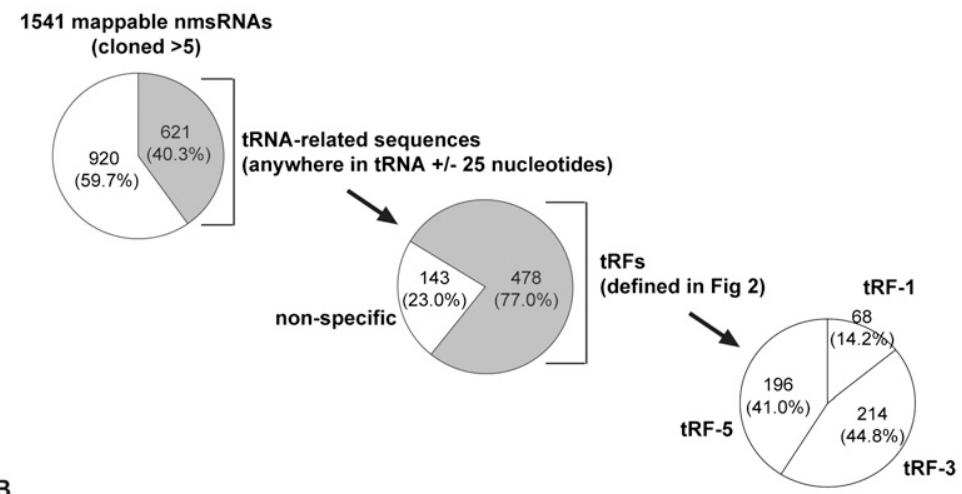

B

mature tRNA

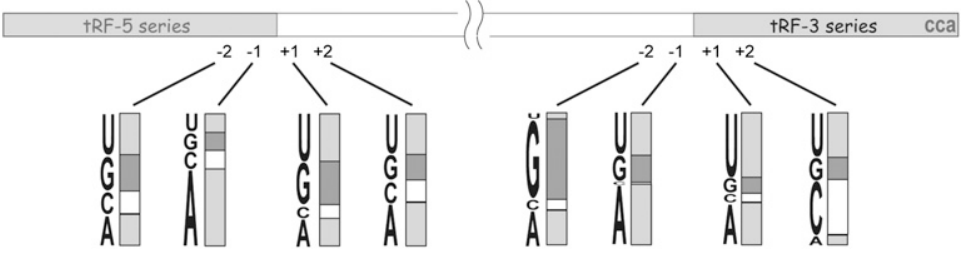

Figure 3. Comprehensive analyses of tRFs: fraction of tRFs in nmsRNAs and characterization of cleavage sites for tRF-5 and tRF-3 series. (A) Among nmsRNAs that were cloned more than five times and mappable in the human genome, tRNA-related sequences /defined as sequences matching anywhere in mature tRNA sequence plus the flanking $25 \mathrm{nt}$ at both ends) were counted and depicted in a pie chart shown at left. In the other two pie charts, tRNA-related sequences were further sorted into the three tRFs (defined and described in the text and Fig. 2B,C) or the rest ("nonspecific $\left.^{\prime \prime}\right) .(B)$ Nucleotide composition at cleavage sites to generate tRF-5 and tRF-3 series. Nucleotides at a given position (numbered in the diagram) in tRFs were counted and normalized to the nucleotide counts of the relative positions of all 622 tRNAs (see also the legend for Supplemental Table S1). The height of each letter (A, C, G, and U) of stacked columns is proportional to the relative frequency of the nucleotide (see Supplemental Table S1 for actual values).

the RNA of 17-26 nt prior to cDNA synthesis to exclude contaminating signals from larger mature tRNAs or other RNAs. qRT-PCR for 12 tRFs successfully detected all of them (Supplemental Fig. S5).

Interestingly, each tRF exhibited a characteristic expression pattern across the cell lines (Supplemental Figs. S4A-C, S5), arguing against the possibility that the tRFs are random by-products from nonspecific degradation of tRNA. Such nonspecific degradation would have resulted in a similar pattern of expression of all tRFs amongst the RNA preparations.

\section{Expression of $t R F-1001$}

tRF-1001 was chosen for further study, as it is the most abundantly cloned tRF and was clearly detectable by Northern hybridization. tRF-1001 is expressed more abundantly in cell lines than in tissues (Fig. 4A, top panel). Despite the greater degradation of RNA from tissues, as indicated by the ethidium bromide (EtBr) staining (Fig. 4A, bottom panel), tRF-1001 levels are not increased in the tissues, reinforcing the notion that tRF1001 is not a by-product of nonspecific RNA degradation.

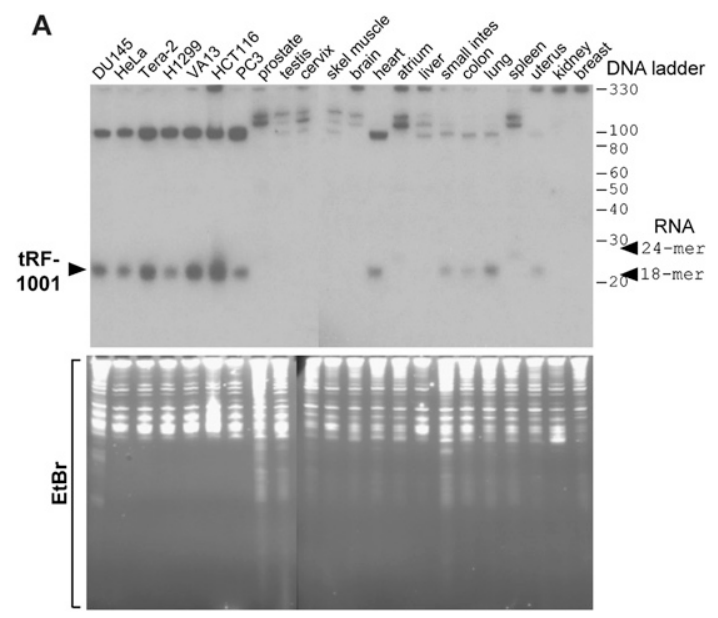

B

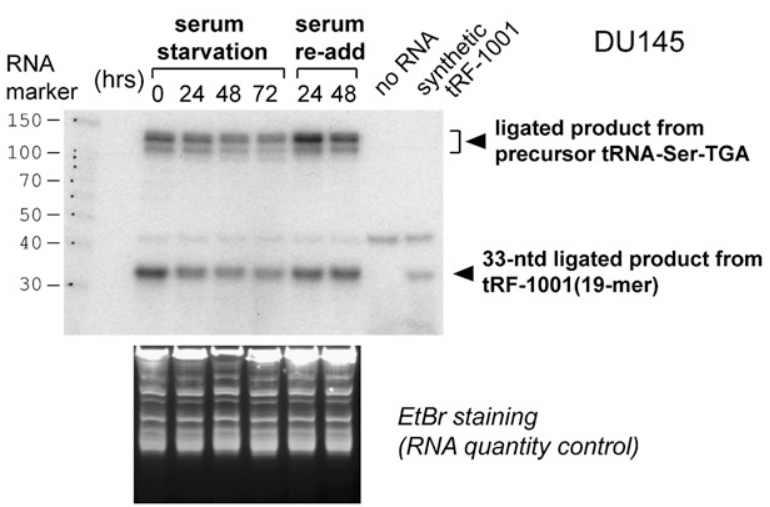

Figure 4. tRF-1001 is highly expressed in proliferating cancer cells. (A, top panel) Northern hybridization of tRF-1001. (Bottom panel) The same amounts of RNA were run in a separate gel for EtBr staining. Source of tissue RNAs is described in Kim et al. (2006). tRF-1001 (19-mer) is indicated by an arrowhead on the left. The positions of 18-and 24-mer synthetic oligoribonucleotides (arrowheads on the right), along with a 10-base-pair DNA ladder, are shown as molecular size markers. (B) Measurement of tRF-1001 and its pre-tRNA upon serum depletion in DU145 cell line. At $72 \mathrm{~h}$ after serum starvation (see the Supplemental Material), DU145 cells were replenished with 10\% FBS medium ("re-add"). Splinted ligation assay of tRF-1001 (top panel) and EtBr staining of total RNA for equal loading (bottom panel). 
The higher expression of tRF-1001 in cancer cell lines of many different lineages suggests that tRF-1001 expression may be related to cell proliferation.

Consistent with this, tRF-1001, measured by splinted ligation or Northerns, is decreased upon serum starvation of DU145 prostate cancer, LNCaP prostate cancer, and HCT116 colon cancer cell lines, and is restored upon serum readdition (Fig. 4B; Supplemental Fig. S6A-C). tRF1001 levels are also reduced when cell density is very high (Supplemental Fig. S6D). Therefore, conditions of poor cell proliferation, as with serum depletion or increased cell density, are associated with a down-regulation of tRF1001. The similar decrease of the precursor tRNA for tRF1001 (Fig. 4B) indicates that the reduction of tRF-1001 upon serum depletion is a result of the down-regulation of the pre-tRNA transcription according to a reduction in general cellular metabolism. In contrast, the corresponding mature tRNA level stayed unchanged (Supplemental Fig. S6A,C,D).

\section{tRF-1001 is required for cell proliferation}

The best argument against the tRFs being a nonspecific byproduct of tRNA metabolism is to demonstrate a functional role for at least one of the tRFs. To investigate this, we knocked down tRF-1001 through an siRNA strategy. We designed three siRNA duplexes that target the pretRNA such that they anneal to part of the tRF-1001 and the mature tRNA (without the CCA). si-tRF1001 is centered at the $+2 /+3$ position of tRF-1001, and two other siRNAs (si-tRF-1001-2 and si-tRF-1001-3) are designed at 1 and $2 \mathrm{nt}$ downstream, respectively (Fig. 5A). Thus, if the siRNAs cleave the pre-tRNA at the center of the siRNA target sequence (Elbashir et al. 2001b), they would cut the pre-tRNA at a site (arrowheads in Fig 5A, in the case of sitRF1001) incompatible with tRF-1001 generation. Alternatively, the siRNAs could interfere with the cleavage of the pre-tRNA by steric interference or by sequestration of the pre-tRNA in RNA-induced silencing complexes (RISCs) (see below).

Cross-hybridization to the passenger strand of sitRF1001 that shares $12 \mathrm{nt}$ with tRF-1001 (and ends with two dTs) obscures the detection of tRF-1001 or its precursor in Northerns or splinted ligations (data not shown). qRT-PCR assay of tRF-1001, however, is not interfered with by the passenger strand of si-tRF1001 for the following reasons. First, the two dTs at the $3^{\prime}$ end of the passenger strand of si-tRF1001 blocks the first step in qRT-PCR, where the polyadenylation of the small RNA by poly(A) polymerase requires $3^{\prime}$ ribonucleotides (Zhelkovsky et al. 1998). Second, the $3^{\prime}$ end of the specific primer used in the second step of qRT-PCR anneals to the tRF but not to the passenger strand of si-tRF1001. Indeed, addition of sitRF1001 during qRT-PCR did not affect the tRF-1001 measurement (data not shown). The reliability of qRTPCR in measuring tRF-1001 was demonstrated by side-byside comparison with Northern hybridization (Supplemental Fig. S7).

qRT-PCR assay confirmed the reduction of tRF-1001 by si-tRF1001 (Supplemental Fig. S8A). In addition to standard qRT-PCR procedure for tRFs using gel-isolated small RNA, qRT-PCR with total RNA yielded a similar result (Supplemental Fig. S8A, right panel). Interestingly, the pre-tRNA level was unchanged by the si-tRF1001 (Supplemental Fig. S8B), suggesting that the decrease in tRF-1001 may be through mechanisms other than cleavage of the pre-tRNA.

Upon tRF-1001 depletion, cell proliferation was impaired, as indicated by a decrease in the number of viable cells (MTT assay in Fig. 5B,D,E; Supplemental Figs. S10, S11), which was accompanied by a reduction of DNA synthesis (bromodeoxyuridine [BrdU] incorporation assay in Fig. 5B) and an accumulation of cells in the G2 phase of the cell cycle (FACS profile in Fig. 5C; Supplemental Fig. S9). Although the si-tRF1001 is expected to decrease the maturation of the tRNA associated with tRF-1001, the same tRNA (Ser-TGA) is expressed from several alternate genomic loci different from the tRF-1001 locus. Indeed, Ser-TGA tRNA level is unchanged after si-tRF1001 treatment (Supplemental Fig. S8B). Thus, the cell proliferation defects induced by si-tRF1001 cannot be attributed to a decrease in the corresponding mature tRNA. Transfection of two other siRNAs (si-tRF1001-2 and sitRF1001-3) (see Fig. 5A for sequence) yielded similar results (Fig. 5D).

Most importantly, the decrease in cell proliferation and G2 accumulation induced by si-tRF1001 were rescued by cotransfection of a synthetic tRF-1001 in the form of a 2'-O-methyl oligoribonucleotide (Me-tRF-1001) (Fig. 5B-E; Supplemental Figs. S9, S10), but not by a similar sequence from an irrelevant gene, luciferase (Me-GL2). This result excludes the possibility that the growth inhibition upon si-tRF1001 transfection is due entirely to a reduction in the pre-tRNA-Ser-TGA or an off-target activity of the siRNA duplex. Instead of a plain oligoribonucleotide that did not rescue in our experimental condition, we used 2 '-O-methyl derivatives, because this modification is known to make the oligoribonucleotide chemically stable and resistant to cellular nucleases (for review, see Lamond and Sproat 1993).

A mutation in either the $5^{\prime}$ or $3^{\prime}$ portion of tRF-1001 resulted in partial rescue, indicating both portions contribute to tRF-1001 function (Fig. 5E). Native tRF-1001 has a phosphate and a hydroxyl group at the $5^{\prime}$ and $3^{\prime}$ ends, respectively, since our cloning protocol depends on this end structure. Both $5^{\prime}$-phosphate and $3^{\prime}$-hydroxyl groups are important for the tRF-1001 function, as the synthetic tRF-1001 lost its capacity to rescue cell proliferation when either the $5^{\prime}$ or the $3^{\prime}$ end is chemically blocked (Supplemental Fig. S10).

Because the sequence of Me-tRF-1001 overlaps the siRNAs by 12-14 nt (Fig. 5A; Supplemental Fig. S11), we wanted to eliminate the possibility that the rescue was because the Me-tRF-1001 anneals to the siRNA and titrates it away from a nonspecific cellular target. Several lines of observation in our experiments assure us of this point. First, the end-blocked Me-tRF-1001 failed to rescue, even though its hybridization capacity is the same as the rescuing oligonucleotides (Supplemental Fig. S10). Second, si-tRF1001-3 produced a more profound effect on 
A

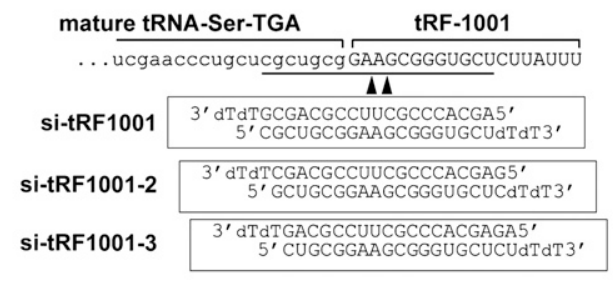

B

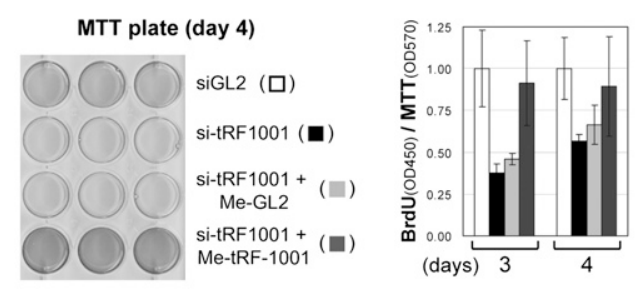

D
C
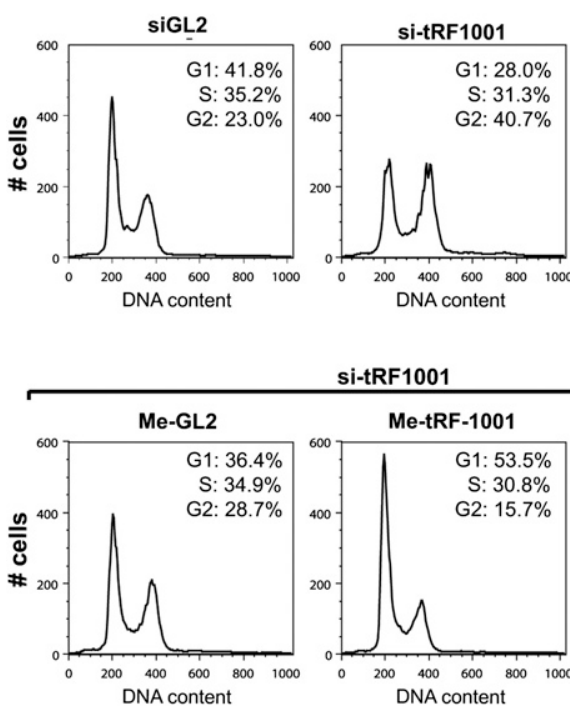

si-tRF1001

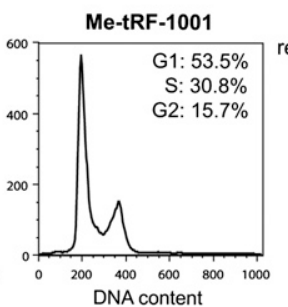

si-tRF1001 relative to siGL2

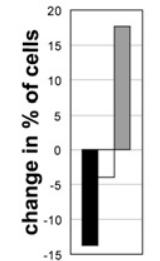

- G1 口 $\square$ G2

Me-tRF-1001 elative to Me-GL2
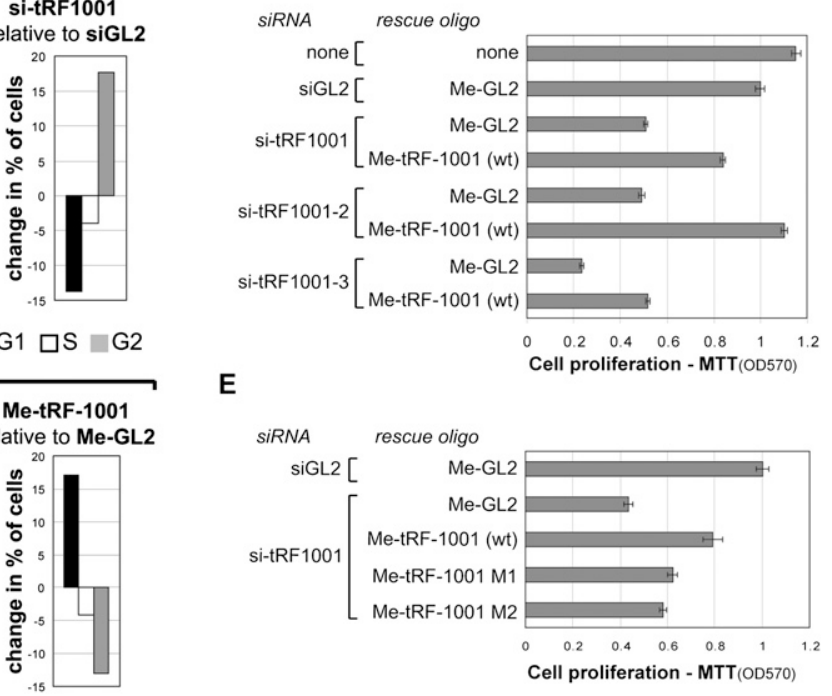

E

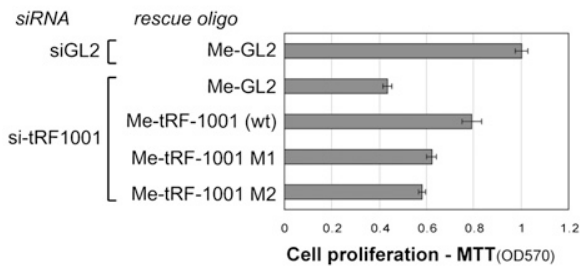

Figure 5. The impaired growth of HCT116 cells upon siRNA-mediated knockdown of tRF-1001 is rescued by Me-tRF-1001. (A) The sequences of siRNA duplexes are shown in alignment with the pre-tRNA sequence of tRF-1001. si-tRF1001 was designed against the underlined region of pre-tRNA, encompassing tRF-1001 (capital letters) and the mature tRNA (lowercase letters). Arrowheads indicate expected sites in the pre-tRNA that will be cleaved by the si-tRF1001 (Elbashir et al. 2001b). (B) MTT and BrdU incorporation assays performed after two rounds of transfection (days 0 and 2) of siGL2 (plain bar), si-tRF1001 (black bar), and si-tRF1001 with Me-GL2 (lightgray bar) or Me-tRF-1001 (dark-gray bar). (Left panel) A scan of wells after MTT assay at day 4. (Right panel) BrdU incorporation normalized to MTT value at days 3 and 4. Average and standard deviation from triplicates are shown. The BrdU/MTT for cells transfected with siGL2 is set as 1. (C) FACS of HCT116 cells transfected (described in B), harvested at day 4, and stained with propidium iodide for DNA content. Cells in G1, S, or G2 were quantitated by ModFit LT for Mac (version 3.2.1). (Right panels) Change in percentage of cells in each phase of the cell cycle between the indicated two samples. $(D)$ MTT assays upon transfection of indicated siRNAs and Me-oligos. After two rounds (days 0 and 1) of transfection of indicated oligonucleotides, MTT assays were performed at day 3. Average and standard deviation from tetraplicates are shown. The value of siGL2 plus Me-GL2 is set as 1 . (E) MTT assays upon cotransfection of tRF-1001 mutants. The sequences of Me-tRF-1001 M1 and M2 are 5'-GCCUAGGGUGCUCUUAUUU-3' and 5'-GAAGCGGGUGCUCAAUAUU-3' (mutated portion italicized), respectively. All other descriptions are same as in $D$.

cell proliferation, which was not fully rescued by Me-tRF1001 (Fig. 5D). Yet, Me-tRF-1001 overlaps more with sitRF-1001-3 than the other two siRNAs and would be expected to better titrate the former by simple hybridization. In addition, we used an Me-control oligoribonucleotide (Supplemental Fig. S11A) that overlaps with another 12 nt of si-tRF1001, but is different from the tRF-1001 sequence. The Me-control oligonucleotide did not rescue the growth impairment induced by si-tRF1001 (Supplemental Fig. S11B), also suggesting that the rescue of cell proliferation by Me-tRF-1001 was not due to titration of the si-tRF1001.

We conclude that tRF-1001 is necessary for cell proliferation. tRF-1001 does not seem to be rate-limiting in usual growth conditions, as transfection of Me-tRF-1001 in untreated cells did not enhance cell proliferation (data not shown).

\section{tRF-1001 is generated by ELAC2 in the cytoplasm}

tRF-1001, typical of tRF-1 molecules, starts precisely after the $3^{\prime}$ end of the mature tRNA before CCA addition (Fig. 2B,C). The $3^{\prime}$ ends of tRF-1 molecules fall within DNA sequences of five to six consecutive Ts (Fig. 2C), a canonical termination site of RNA polymerase III transcription (Hagenbuchle et al. 1979; Koski and Clarkson 1982; Cozzarelli et al. 1983). This sequence structure suggests that the tRF-1 series of small RNAs are 3 '-trailer sequences from pre-tRNA, released through a cleavage by tRNA endonuclease ELAC2 during the 3 '-end maturation 
Lee et al.

of tRNA (Takaku et al. 2003). Consistent with this scenario, knockdown of ELAC2 resulted in a reduction of tRF-1001 and a concomitant accumulation of the pretRNA (Fig. 6A). tRF-1001 and its precursor tRNA are exclusively localized in the cytoplasm, suggesting that the biogenesis of tRF-1001 occurs in the cytoplasm (Fig. $6 \mathrm{~B}, \mathrm{C})$.

The size (19-mer) and cytoplasmic localization of tRF1001 raise the possibility that tRF-1001 may incorporate into the siRNA or miRNA pathway. To address this question, we performed an miRNA/siRNA assay using a sensor plasmid. This plasmid contains a perfect antisense to tRF-1001 downstream from the luciferase, and therefore would respond to tRF-1001 if the latter acted as an siRNA or miRNA. There was no change in the luciferase reporter expression upon modulation of the intracellular level of tRF-1001 through serum deprivation or transfection of si-tRF1001 or Me-tRF-1001 (Supplemental Fig. S12 and its legend). Thus, tRF-1001 is functionally distinct from miRNA/siRNA.

In summary, our data show that tRF-1001 is a novel small RNA that is generated by ELAC2 from cleavage of the pre-tRNA in the cytoplasm, is regulated according to cell proliferation, and is necessary for cell viability (Fig. 6D).

\section{Discussion}

We report that tRFs are a novel class of small RNAs that are second in abundance only to miRNAs. tRF-5 and tRF-3 are derived from mature tRNAs. Although it is conceivable that as many of these tRFs will exist as there are mature tRNAs, we did not clone all of the possible tRF-5 and tRF-3. Whether this is due to our limited cloning and sequencing effort or due to biological differences in the processing of tRNAs will be determined as we accumulate more reports of cloning and sequencing of short RNAs. tRF-1 series members are 3 '-trailer sequences in pre-tRNA. Of the 622 tRNA loci, an analysis of the distance between the $3^{\prime}$ end of the mature tRNA and $3^{\prime}$-termination site of the pre-tRNA (Supplemental Fig. S3) indicates that fewer tRNA loci can produce a tRF-1 series of $17-26 \mathrm{nt}$. However, it is entirely possible that future cloning of longer or shorter RNAs will yield more tRF-1 clones. Like miRNAs, we expect different sets of tRFs to be expressed in different cell lines and tissues under different growth conditions, and future efforts will be directed at expanding the repertoire of tRFs.

Recent reports are beginning to include tRNA cleavage as a mechanism of biogenesis of small RNAs. tRNA fragments of 30-35 nt have been identified in bacteria, fungi, plants, and animals (Lee and Collins 2005; Haiser et al. 2008; Jochl et al. 2008; Kawaji et al. 2008; Li et al. 2008; Thompson et al. 2008; Zhang et al. 2009). These tRNA fragments are different from the ones reported here in that they are derived from a cleavage at or around the anti-codon loop by Rnylp in yeast (Thompson and Parker 2009) or angiogenin in humans (Yamasaki et al. 2009). In addition, although Rnylp and angiogenin promoted stress-induced cell death and translational repression,
Figure 6. tRF-1001 is generated by ELAC2 in the cytoplasm. (A) DU145 cells were transfected with an siRNA against ELAC2. (Top two panels) Knockdown of ELAC2 was confirmed by Western blot, with $\beta$-actin as a loading control. tRF-1001 and its pre-tRNA was measured by splinted ligation assays (middle two panels), with EtBr staining of total RNA (bottom panel) as a loading control. (B) Total, nuclear, and cytoplasmic RNAs from LNCaP cells were subjected to Northern hybridization of tRF-1001, snoU38 as a nuclear marker, and $5 \mathrm{~S}$ rRNA and EtBr staining of total RNA as a loading control. $(C)$ Measurement of tRF-1001 in total, nuclear, and cytoplasmic RNAs from DU145 cells. EtBr staining of total RNA is shown as both quantity and quality control (compare EtBr staining pattern of fractionated RNAs in $B$ ). (D) A model for biogenesis and regulation of tRF-1001. See the text for details.
A

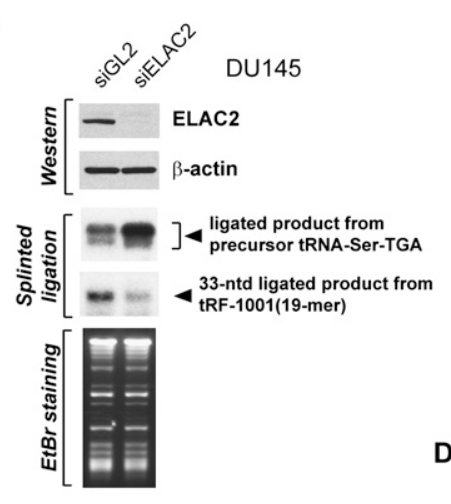

B

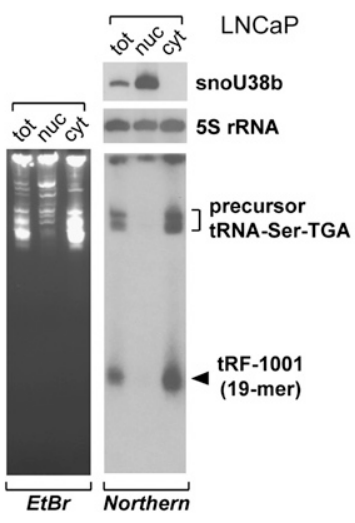

D
C
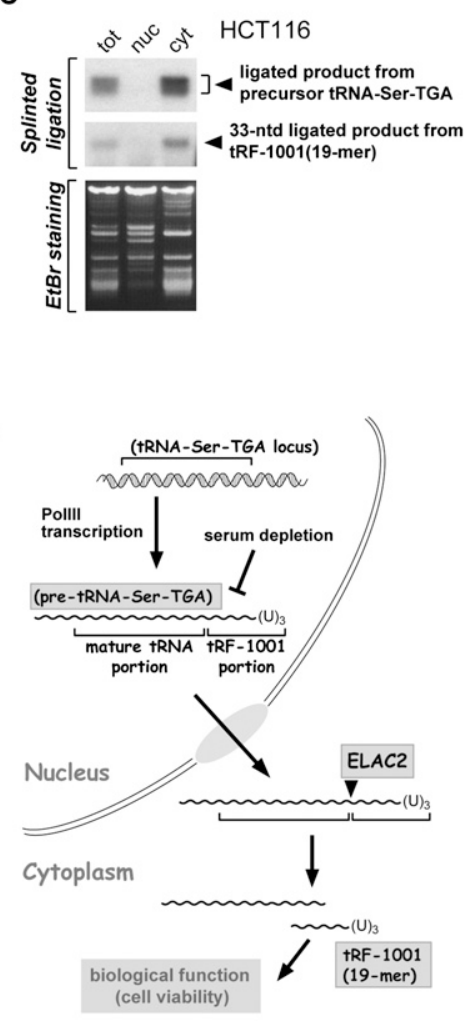
respectively, there was no evidence that a specific tRNA fragment was responsible for either of these phenotypes. tRF-1001 is the first tRNA-derived short RNA whose depletion is shown to produce a specific biological phenotype.

Biogenesis of tRF-1001, and most likely all short RNAs of the tRF-1 series, is catalyzed by ELAC2, which had been identified originally as a candidate prostate cancer susceptibility gene (Tavtigian et al. 2001) and was revealed later as an endonuclease for $3^{\prime}$ trimming of pre-tRNA (Takaku et al. 2003). To our surprise, tRF1001 as well as its pre-tRNA was detected exclusively in the cytoplasm. It is believed that $5^{\prime}$ and $3^{\prime}$ trimming of pre-tRNA to produce the mature tRNA occurs in the nucleus (for review, see Hopper and Shaheen 2008). We speculate that pre-tRNA transcripts from a given tRNA gene may have two fates: rapid cleavage in the nucleus for biogenesis of mature tRNA, or export to the cytoplasm for generation of tRF-1 series of short RNA. The pretRNA for tRF-1001 was not detected in the nucleus (Fig. $6 \mathrm{~B}, \mathrm{C})$, possibly because processing to mature tRNA or export to the cytoplasm is too fast to detect a steady-state level of nuclear pre-tRNA. In contrast, the cytoplasmically exported pre-tRNA that serves to generate tRF-1001 is more stable and can be detected easily. A cytoplasmic location of the tRF-1001 precursor pre-tRNA is consistent with its susceptibility to siRNA, since the siRNA machinery is believed to poorly target nuclear RNAs. Interestingly, ELAC2, the enzyme that trims the pretRNA to generate tRF-1001, is localized predominantly in the cytoplasm (Korver et al. 2003).

The mechanism of biogenesis of tRF- 5 and tRF- 3 series is not clear yet. Most likely, they are generated from cleavage of mature tRNA by specific nucleases, as indicated by the juxtaposition of the start and end points of tRFs with those of mature tRNAs. Sequence analyses of cleavage sites (Fig. 3B) suggest that the tRF-5 series are preferentially cleaved after A and the tRF-3 series between $\mathrm{A} / \mathrm{U}$ and $\mathrm{A} / \mathrm{U}$. Several tRNA-specific nucleases, such as Colicin E5, Colicin D, PrrC (for review, see Saida and Odaert 2007), Ranpirnase or Onconase (Ardelt et al. 1991; Saxena et al. 2002), and angiogenin (Fett et al. 1985), have been reported from various organisms. However, the preferential cleavage sites are not explained well by any of these enzymes. Cleavage sites of tRF- 5 and tRF-3 series are mapped within the $\mathrm{D}$ arm and the $\mathrm{T} \psi \mathrm{C}$ arm, respectively. However, we could not find any consensus feature of cleavage sites on the cloverleaf secondary structure of tRNA. Among the studies reporting tRNA fragments, Zhang et al. (2009) reported $\sim 58$ - to 60-ntlong fragments whose cleavage sites fall in the D arm. These fragments could represent the reciprocal fragments generated upon release of tRF-5 series.

Intriguingly, the size distribution of tRF-3 series exhibits a skewed distribution toward a smaller size range, with significant depletion at a size of $20 \mathrm{nt}$ (Supplemental Fig. S2). This depletion may be explained by inefficient cleavage at the -20 position (from the $3^{\prime}$ end of mature tRNA with CCA), possibly because the nucleotide at the -21 position of mature tRNA is mostly $\mathrm{C}(91 \%)$ (see also
Fig 3B). Another possibility is that a base modification at the site may confer resistance to cleavage. However, no prominent modification was reported in the $-19,-20$, and -21 positions (Grosjean et al. 1995).

The tRF-1 series are of particular interest, not only because we show that one of them is required for cell proliferation, but also because the sequence downstream from the tRNA that is encoded in the pre-tRNA is mostly unique among different tRNA genes (Supplemental Table S2). Mature tRNAs of identical or highly similar sequences are repeated at multiple loci in the genome, with 622 tRNA loci coding for tRNAs containing, at most, 64 anticodons. We speculate that some tRNA genes have additional gene-specific functions by generating unique tRFs from the 3 '-flanking sequences, perhaps explaining the duplication and survival of so many tRNA genes during evolution.

Much more work needs to be done to investigate how many tRFs are expressed, what regulates their levels, how many have discernible biological roles, and whether the tRFs exert their biological functions as part of ribonucleoprotein complexes. Despite this, however, it is exciting to consider the obvious parallels between the current status of tRFs with what was known about miRNAs soon after it became evident that hundreds of different miRNAs are present in mammalian cells (Lagos-Quintana et al. 2001; Lau et al. 2001; Lee and Ambros 2001).

\section{Materials and methods}

Isolation of RNA, 454 deep sequencing, and measurement of $t R F s$

Isolation of total, nuclear, and cytoplasmic RNA from cell lines was performed as described in Lee and Dutta (2007). Small RNAs of 17-26 nt were cloned and subjected to 454 deep sequencing. The probe sequences for Northerns are $5^{\prime}$-AAATAAGAGCACC CGCTTC-3' (tRF-1001), 5'-GATCGGGCGCGTTCAGGGTGG TAT-3' (5S rRNA), and 5'-AGAACTGGACAAAGTTTTCAT CAC-3' (snoU38b, a snoRNA). Splinted ligation assays were performed as described in Maroney et al. (2008). The oligonucleotides 5'-CGCTTATGACATTCC-3' (ligation oligo) and 5'-GAAT GTCATAAGCGAAATAAGAGCACCCGCTTC-3' (bridge oligo) are designed to harbor modifications as described (Maroney et al. 2008).

\section{Cell culture and transfection of RNA oligonucleotides}

Cell lines and culture conditions are described in the Supplemental Material. siRNA duplexes were synthesized by Invitrogen Corp. The sequences of siRNAs against tRF-1001 are shown in Figure 5A, siGL2 sequence is described in Elbashir et al. (2001a), and siRNA against ELAC2 targets 5'-GUGUCCGAGA CUUAACGAA-3' . 2'-O-methyl-modified GL2 (5'-CGUACGCG GAAUACUUCGA-3'), tRF-1001 (see Fig. 5A for sequence), and its mutants (see the legend for Fig. 5E for the sequences) were synthesized by Dharmacon RNA Technologies. Transfection of short RNA was performed with Lipofectamine RNAiMAX reagent (Invitrogen Corp.) using $33 \mathrm{nM}$ siRNA duplex and $67 \mathrm{nM}$ 2'-O-methyl oligonucleotide, according to the reverse transfection procedure in the manufacturer's instructions. The first transfection (day 0) into HCT116 cells was followed by a second transfection at day 1 or 2 . Total RNA was isolated and various 
assays were performed at day 4, unless otherwise indicated. During the course of the transfection experiment, HCT116 cells were kept below a density of $2 \times 10^{7}$ cells per $10-\mathrm{cm}$ culture dish.

\section{Measurement of cell proliferation and DNA synthesis}

Cell proliferation was measured with CellTiter 96 nonradioactive cell proliferation assay kit (MTT assay; Promega Corp.). DNA synthesis was assayed by BrdU ELISA as described in Machida et al. (2006). All experiments were performed in triplicates or tetraplicates, from which average and standard deviation were calculated and plotted.

\section{Acknowledgements}

This work was supported by a grant from the Department of Defense Prostate Cancer Research Program, IDEA PC050114 to A.D.

\section{References}

Ardelt W, Mikulski SM, Shogen K. 1991. Amino acid sequence of an anti-tumor protein from Rana pipiens oocytes and early embryos. Homology to pancreatic ribonucleases. I Biol Chem 266: 245-251.

Bartel DP. 2004. MicroRNAs: Genomics, biogenesis, mechanism, and function. Cell 116: 281-297.

Chapman EJ, Carrington JC. 2007. Specialization and evolution of endogenous small RNA pathways. Nat Rev Genet 8: 884896.

Cozzarelli NR, Gerrard SP, Schlissel M, Brown DD, Bogenhagen DF. 1983. Purified RNA polymerase III accurately and efficiently terminates transcription of 5S RNA genes. Cell 34: 829-835.

Du T, Zamore PD. 2005. microPrimer: The biogenesis and function of microRNA. Development 132: 4645-4652.

Elbashir SM, Harborth J, Lendeckel W, Yalcin A, Weber K, Tuschl T. 2001a. Duplexes of 21-nucleotide RNAs mediate RNA interference in cultured mammalian cells. Nature 411: 494-498.

Elbashir SM, Lendeckel W, Tuschl T. 2001b. RNA interference is mediated by 21- and 22-nucleotide RNAs. Genes \& Dev 15: 188-200.

Farazi TA, Juranek SA, Tuschl T. 2008. The growing catalog of small RNAs and their association with distinct Argonaute/ Piwi family members. Development 135: 1201-1214.

Fett JW, Strydom DJ, Lobb RR, Alderman EM, Bethune JL, Riordan JF, Vallee BL. 1985. Isolation and characterization of angiogenin, an angiogenic protein from human carcinoma cells. Biochemistry 24: $5480-5486$.

Giege R. 2008. Toward a more complete view of tRNA biology. Nat Struct Mol Biol 15: 1007-1014.

Grosjean H, Sprinzl M, Steinberg S. 1995. Posttranscriptionally modified nucleosides in transfer RNA: Their locations and frequencies. Biochimie 77: 139-141.

Hagenbuchle O, Larson D, Hall GI, Sprague KU. 1979. The primary transcription product of a silkworm alanine tRNA gene: Identification of in vitro sites of initiation, termination and processing. Cell 18: 1217-1229.

Haiser HJ, Karginov FV, Hannon GJ, Elliot MA. 2008. Developmentally regulated cleavage of tRNAs in the bacterium Streptomyces coelicolor. Nucleic Acids Res 36: 732-741.

Hopper AK, Phizicky EM. 2003. tRNA transfers to the limelight. Genes \& Dev 17: 162-180.
Hopper AK, Shaheen HH. 2008. A decade of surprises for tRNA nuclear-cytoplasmic dynamics. Trends Cell Biol 18: 98-104.

Jochl C, Rederstorff M, Hertel J, Stadler PF, Hofacker IL, Schrettl M, Haas H, Huttenhofer A. 2008. Small ncRNA transcriptome analysis from Aspergillus fumigatus suggests a novel mechanism for regulation of protein synthesis. Nucleic Acids Res 36: 2677-2689.

Kapranov P, Cheng J, Dike S, Nix DA, Duttagupta R, Willingham AT, Stadler PF, Hertel J, Hackermuller J, Hofacker IL, et al. 2007. RNA maps reveal new RNA classes and a possible function for pervasive transcription. Science 316: 1484-1488.

Kawaji H, Nakamura M, Takahashi Y, Sandelin A, Katayama S, Fukuda S, Daub CO, Kai C, Kawai J, Yasuda J, et al. 2008. Hidden layers of human small RNAs. BMC Genomics 9: 157. doi: 10.1186/1471-2164-9-157.

Kim HK, Lee YS, Sivaprasad U, Malhotra A, Dutta A. 2006. Muscle-specific microRNA miR-206 promotes muscle differentiation. J Cell Biol 174: 677-687.

Korver W, Guevara C, Chen Y, Neuteboom S, Bookstein R, Tavtigian S, Lees E. 2003. The product of the candidate prostate cancer susceptibility gene ELAC2 interacts with the $\gamma$-tubulin complex. Int I Cancer 104: 283-288.

Koski RA, Clarkson SG. 1982. Synthesis and maturation of Xenopus laevis methionine tRNA gene transcripts in homologous cell-free extracts. J Biol Chem 257: 4514-4521.

Lagos-Quintana M, Rauhut R, Lendeckel W, Tuschl T. 2001. Identification of novel genes coding for small expressed RNAs. Science 294: 853-858.

Lamond AI, Sproat BS. 1993. Antisense oligonucleotides made of 2'-O-alkylRNA: Their properties and applications in RNA biochemistry. FEBS Lett 325: 123-127.

Lau NC, Lim LP, Weinstein EG, Bartel DP. 2001. An abundant class of tiny RNAs with probable regulatory roles in Caenorhabditis elegans. Science 294: 858-862.

Lee RC, Ambros V. 2001. An extensive class of small RNAs in Caenorhabditis elegans. Science 294: 862-864.

Lee SR, Collins K. 2005. Starvation-induced cleavage of the tRNA anticodon loop in Tetrahymena thermophila. I Biol Chem 280: 42744-42749.

Lee YS, Dutta A. 2007. The tumor suppressor microRNA let-7 represses the HMGA2 oncogene. Genes \& Dev 21: 10251030.

Lee YS, Dutta A. 2009. MicroRNAs in cancer. Annu Rev Pathol 4: 199-227.

Li Y, Luo J, Zhou H, Liao JY, Ma LM, Chen YQ, Qu LH. 2008. Stress-induced tRNA-derived RNAs: A novel class of small RNAs in the primitive eukaryote Giardia lamblia. Nucleic Acids Res 36: 6048-6055.

Machida YJ, Chen Y, Machida Y, Malhotra A, Sarkar S, Dutta A. 2006. Targeted comparative RNA interference analysis reveals differential requirement of genes essential for cell proliferation. Mol Biol Cell 17: 4837-4845.

Maroney PA, Chamnongpol S, Souret F, Nilsen TW. 2008. Direct detection of small RNAs using splinted ligation. Nat Protoc 3: 279-287.

Morl M, Marchfelder A. 2001. The final cut. The importance of tRNA 3'-processing. EMBO Rep 2: 17-20.

Morozova O, Marra MA. 2008. Applications of next-generation sequencing technologies in functional genomics. Genomics 92: $255-264$.

Nilsen TW. 2008. Endo-siRNAs: Yet another layer of complexity in RNA silencing. Nat Struct Mol Biol 15: 546-548.

Saida F, Odaert B. 2007. RNA recognition and cleavage by sequence-specific endoribonucleases. Protein Pept Lett 14: $103-111$. 
Saxena SK, Sirdeshmukh R, Ardelt W, Mikulski SM, Shogen K, Youle RJ. 2002. Entry into cells and selective degradation of tRNAs by a cytotoxic member of the RNase A family. I Biol Chem 277: 15142-15146.

Schmittgen TD, Lee EJ, Jiang J, Sarkar A, Yang L, Elton TS, Chen C. 2008. Real-time PCR quantification of precursor and mature microRNA. Methods 44: 31-38.

Taft RJ, Glazov EA, Cloonan N, Simons C, Stephen S, Faulkner GJ, Lassmann T, Forrest AR, Grimmond SM, Schroder K, et al. 2009. Tiny RNAs associated with transcription start sites in animals. Nat Genet 41: 572-578.

Takaku H, Minagawa A, Takagi M, Nashimoto M. 2003. A candidate prostate cancer susceptibility gene encodes tRNA 3' processing endoribonuclease. Nucleic Acids Res 31: 22722278.

Tavtigian SV, Simard J, Teng DH, Abtin V, Baumgard M, Beck A, Camp NJ, Carillo AR, Chen Y, Dayananth P, et al. 2001. A candidate prostate cancer susceptibility gene at chromosome 17p. Nat Genet 27: 172-180.

Thompson DM, Parker R. 2009. The RNase Rnylp cleaves tRNAs and promotes cell death during oxidative stress in Saccharomyces cerevisiae. J Cell Biol 185: 43-50.

Thompson DM, Lu C, Green PJ, Parker R. 2008. tRNA cleavage is a conserved response to oxidative stress in eukaryotes. RNA 14: 2095-2103.

Yamasaki S, Ivanov P, Hu GF, Anderson P. 2009. Angiogenin cleaves tRNA and promotes stress-induced translational repression. J Cell Biol 185: 35-42.

Zhang S, Sun L, Kragler F. 2009. The phloem-delivered RNA pool contains small noncoding RNAs and interferes with translation. Plant Physiol 150: 378-387.

Zhelkovsky A, Helmling S, Moore C. 1998. Processivity of the Saccharomyces cerevisiae poly(A) polymerase requires interactions at the carboxyl-terminal RNA binding domain. Mol Cell Biol 18: 5942-5951. 


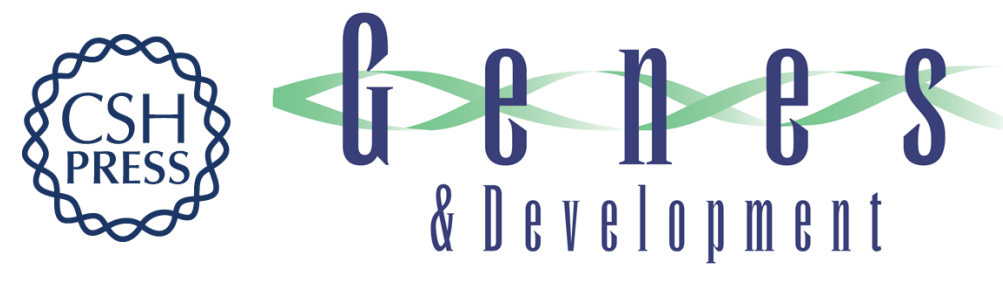

\section{A novel class of small RNAs: tRNA-derived RNA fragments (tRFs)}

Yong Sun Lee, Yoshiyuki Shibata, Ankit Malhotra, et al.

Genes Dev. 2009, 23:

Access the most recent version at doi:10.1101/gad.1837609

Supplemental
Material http://genesdev.cshlp.org/content/suppl/2009/11/19/23.22.2639.DC1

References This article cites 45 articles, 20 of which can be accessed free at: http://genesdev.cshlp.org/content/23/22/2639.full.html\#ref-list-1

License

Email Alerting Receive free email alerts when new articles cite this article - sign up in the box at the top Service right corner of the article or click here.

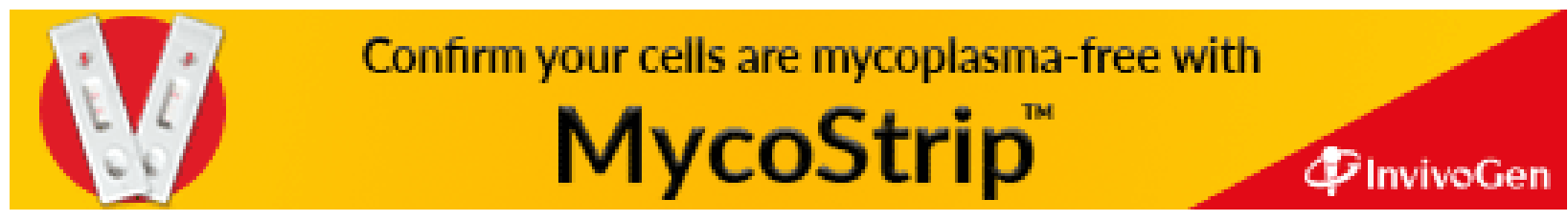

